\title{
Deeds not words needed on shameful oral health inequalities
}

The British Dental Association has called on ministers to act on the latest report from Public Health England on the shocking oral health gap between rich and poor, warning that without dedicated action from government inequalities will widen as a result of COVID-19.

The report shows relative inequalities in the prevalence of tooth decay in 5-year-old children in England increased from 2008 to 2019. The BDA has expressed deep concern these inequalities will widen given the suspension of public health programmes over the last year, and ongoing difficulties accessing services.

Decay remains the number one reason for hospital admissions among young children, with official surveys indicating a tenfold difference in severity of tooth decay between the richest and most deprived communities.

The BDA strongly endorses PHE's policy recommendations on supervised brushing in early years settings and water fluoridation, both of which are clinically proven and costsaving according to government own modelling, based on the corresponding reduction in treatment need.

Dentist leaders have welcomed recent signs of support for both policies from the Department of Health, but stressed progress will only be possible if government is prepared to offer dedicated funding to support set-up and operating costs. The government's promise to consult on a national supervised brushing scheme for England by the end of 2020 has yet to materialise.

BDA Chair Eddie Chair said: 'The oral health gap between rich and poor is not inevitable. Sadly, the pandemic means

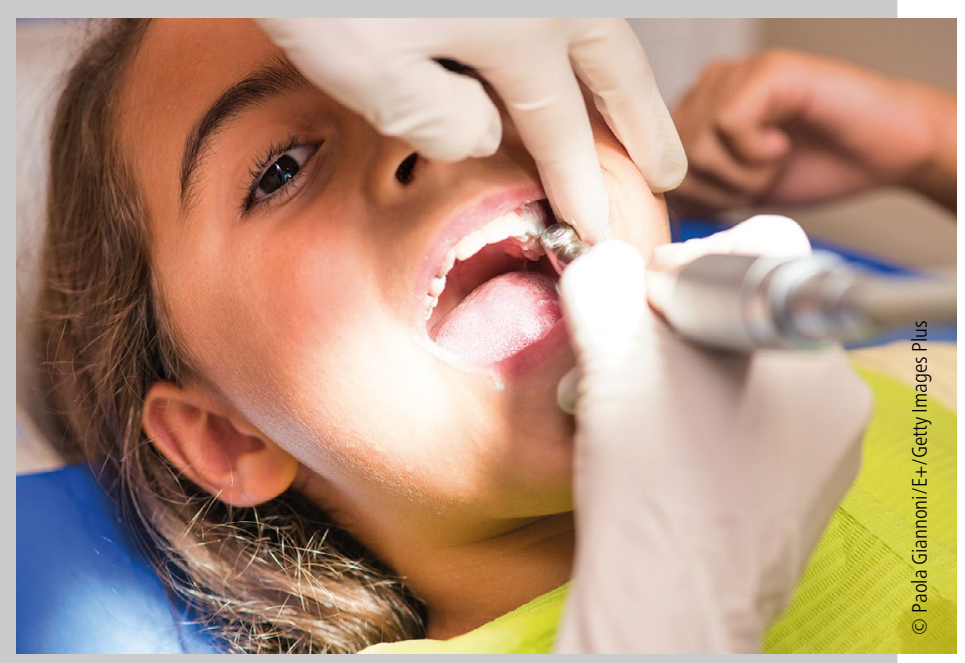

shameful levels of inequality are set to widen.

'We need deeds not words from government, with real support for practices and action on prevention. These programmes can pay for themselves, but until Ministers offer tangible commitments we will not see progress.

\section{NEWS}

\section{GDC introduces option to pay ARF by instalments}

A new pay by instalments option for the Annual Retention Fee (ARF) has been announced by the General Dental Council designed to support dental professionals who pay their own fee by enabling them to spread the cost over the year with quarterly direct debits. For dental care professionals (DCP) this works out as four payments of $£ 28.50$ and for dentists it is four payments of $£ 170$.

To take advantage of the option in 2021, DCPs need to log in to eGDC from early May and select the quarterly direct debit option no later than 31 May 2021. Dentists who wish to pay their ARF by instalments will be able to select this option later in the year.

GDC Chief Executive and Registrar, Ian Brack, said: 'We know that many dental professionals want more flexibility in how they pay the ARF and so we will be introducing a pay by instalments option. Detailed feasibility work has been completed and work is underway to make the necessary changes to our systems to allow us to offer this to DCPs this year.
'We expect this work to be complete by early May and DCPs who want to pay quarterly will then need to sign up by 31 May. Detailed guidance will follow nearer the time.'

Commenting on the news, Shareena Ilyas, Chair of the British Dental Association's Education, Ethics and the Dental Team Working Group said: 'The ability to pay by instalments is one of the basics any registrant should expect from their regulator. This represents much needed change from the GDC, and not before time.

'A single large annual payment has proved a real barrier for manv dentists narticularly those

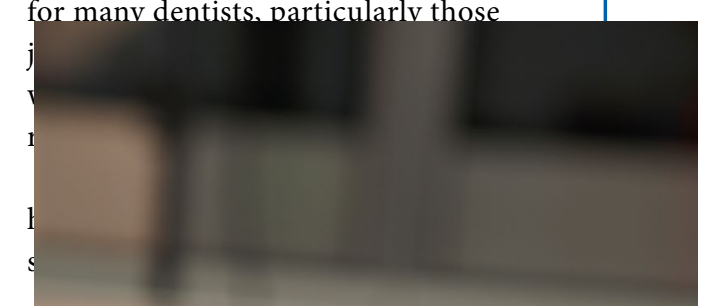

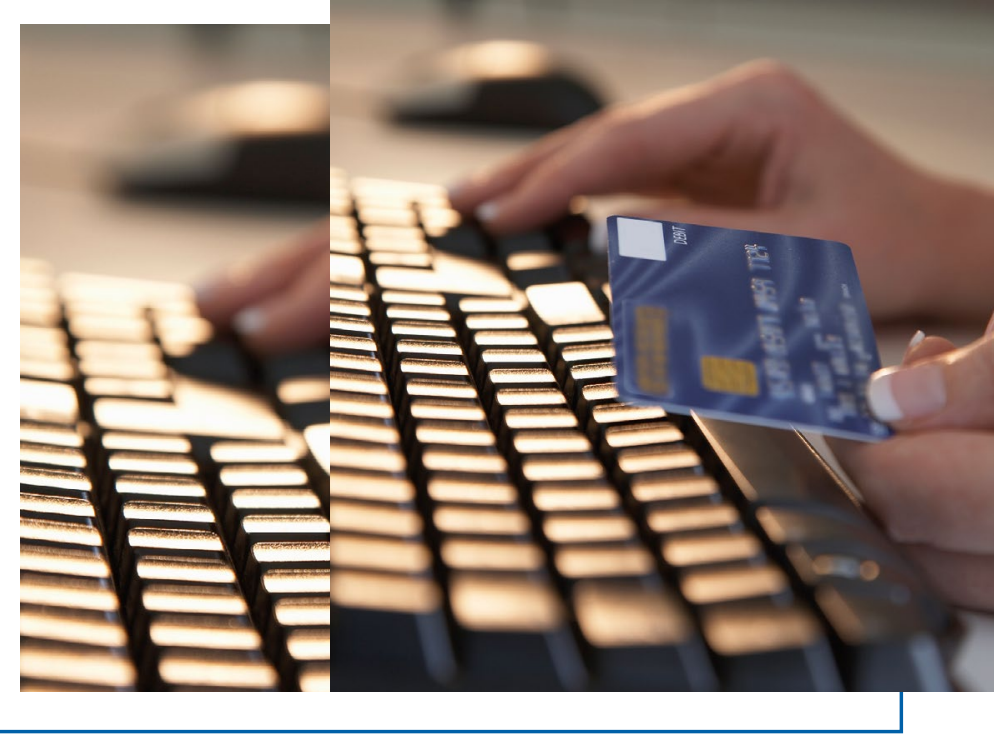

